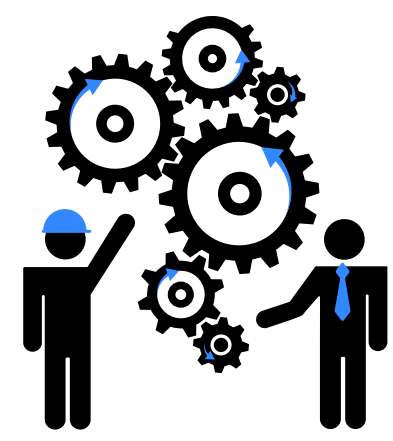

\title{
IMPORTANCIA DEL MÉTODO DE COEFICIENTES DEL ACI EN EL DISEÑO DE LOSAS CONTINUAS DE CONCRETO ARMADO
}

Method importance of ACI coefficients in the design of reinforced concrete continuous slabs

Natividad A. Sánchez Arévalo ${ }^{1}$, Carlos Lermo Zuñiga ${ }^{2}$, Jeysi Ochoa Laguna ${ }^{3}$

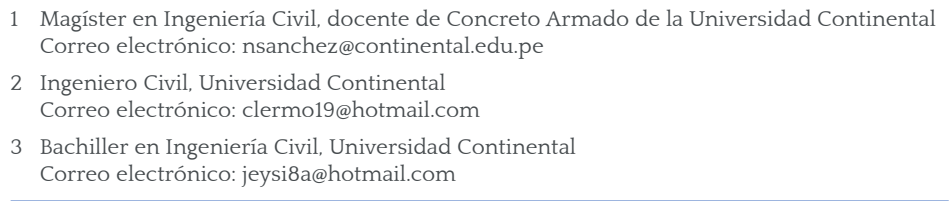

Resumen-El método de los coeficientes del ACI[1] es una herramienta que permite a los ingenieros civiles diseñar, en forma muy simplificada, vigas y losas horizontales que corresponden a sistemas estructurales continuos con apoyos simples y/o muros o columnas, sometidos a cargas de gravedad. Los coeficientes proporcionados por este método son los resultados de considerar las alternancias de cargas en las vigas o losas continuas.

$\mathrm{Al}$ aplicar este método, surge la controversia del tipo de coeficientes que se deben emplear en los apoyos que constituyen muros o columnas, además de no entender la discontinuidad con que se dibujan los diagramas resultantes de momentos flectores. Para aclarar estas dudas y tener la seguridad de que la aplicación de este método es confiable, en el presente artículo se plantea una estructura típica de cuatro tramos, para dos tipos de aligerados: uno con apoyos simples y otro con la combinación de apoyos simples y muros.

Para los dos tipos de losa, se obtienen los diagramas de momentos flectores y se analizan las estructuras de dos maneras: con el método de coeficientes y con el programa SAP 2000, para diferentes configuraciones de luces contiguas y en cada una de ellas para diferentes casos de apoyos y cargas, considerando la alternancia de cargas vivas.

Los resultados del análisis demuestran buena aproximación entre aquellos obtenidos con el método de coeficientes y aquellos obtenidos analíticamente con el programa SAP.

Se concluye que el método del ACI [1] permite trabajar en forma rápida y precisa, con la garantía de obtener resultados confiables porque considera la alternancia de cargas. Siempre y cuando se cumpla con las limitaciones del método.

Palabras clave: Coeficientes ACI; alternancia de cargas; momentos flectores; apoyos continuos y discontinuos.

\begin{abstract}
The ACI coefficient method is a tool that allows the engineer to design, in a rather simplified way, beams and slabs that correspond to continuous structural systems with simple supports and/or walls or columns, subjected to weight charges. The coefficients provided by this method are the results of considering the alternation of charges between the beams or continuous slabs.

By applying this method, the controversy of the nature of coefficients to use on supports that constitute walls or columns may rise, also from misunderstanding the discontinuity with which some bending moments resulting diagrams may be drawn. To clear these doubts and have the security that the application of this method is trustworthy, it is proposed in the present article, a common four-sectioned structure, in which will be present two different kinds of lightened slabs, one with simple support structures and another one with the combination of simple supports and walls.
\end{abstract}

From the mentioned types of slab, the bending moments diagrams are obtained by analyzing the structure in two different ways: With the coefficient method; and with the program SAP 2000, for different configurations and on each one of them for different kinds of supports, spans and charges, considering the alternation of live charges.

The analysis results show good approximation between the ones obtained with the coefficients method and the ones obtained analytically with SAP, considering the alternation of live charges.

In conclusion, the ACI method allows working in a quick and precise manner, with the guarantee of obtaining trusty results since it considers the alternation of charges. As long as the limitations of the method are complied.

Keywords: ACI coefficients, charges alternation, bending moments, continuous and discontinuous support. 


\section{INTRODUCCIÓN}

Los objetivos del presente artículo son:

1) Demostrar que el método de coeficientes proviene de los resultados obtenidos del análisis estructural de vigas continuas con alternancia de cargas vivas.

2) Demostrar que el método de coeficientes tiene resultados muy aproximados a los obtenidos por análisis más exactos, siempre y cuando se cumplan las limitaciones establecidas por el método.

3) Puntualizar los coeficientes empleados para los apoyos extremos cuando estos se tratan de muros o columnas.

Para dicho fin con base en la estructuración de la planta de una losa aligerada de un centro educativo de tres pisos, con dos tipos de aligerados: el aligerado «a», con 3 apoyos muros y 2 apoyos vigas, y el aligerado «b», con la totalidad de apoyos vigas, se desarrollan los siguientes temas:

La metodología utilizada especifica el uso de los métodos de análisis estructural para calcular los momentos flectores de las losas, según los casos planteados en II. Se utiliza el método de coeficientes del ACI y el método de análisis con el programa SAP2000.

Las configuraciones, modelos y análisis estructural comprende:

1. Configuraciones de las losas aligeradas: con luces iguales, con luces contiguas aproximadamente iguales, con luces contiguas desiguales con diferencias superiores al 20\% y con carga viva superior a tres veces la carga muerta.

2. Para las tres primeras configuraciones se han desarrollado los siguientes casos:

- Casos del aligerado a con apoyos combinados:

- Caso 1: Vigas y muro de albañilería artesanal

- Caso 2: Vigas y muro de albañilería Industrial

- Caso 3: Vigas y muro de concreto armado $\mathrm{e}=0.25 \mathrm{~m}$

- Caso 4: Vigas y muro de concreto armado $\mathrm{e}=0.30 \mathrm{~m}$

- Casos del aligerado b

- Caso 5: Todos los apoyos son vigas

Para la última configuración, se ha desarrollado solo el caso que corresponde al aligerado «b» con todos los apoyos simples.

\section{METODOLOGIA UTILIZADA}

Los métodos utilizados para el logro de los objetivos de este artículo son 1) el método de coeficientes del ACI y 2) el método analítico, con la utilización del programa SAP 2000.

\section{El método de coeficientes del ACI[1]}

Este método, reconocido, por la Norma Técnica de Concreto Armado E 0.60 [2], permite calcular momentos y fuerzas cortantes para el diseño de elementos continuos.

Se puede utilizar como alternativa al análisis estructural de pórticos continuos para cargas de gravedad.
Las limitaciones de este método son:

- Vigas continuas, losas aligeradas o losas armadas en una dirección.

- Deben de haber dos o más tramos de luces más o menos iguales. La luz del tramo mayor, de dos adyacentes, no debe exceder en más del $20 \%$ de la luz del menor (L mayor $\leq 1.2 \mathrm{~L}$ menor).

. Elementos prismáticos de sección constante.

- La carga viva no debe exceder de tres veces la carga muerta.

- Solo debe haber cargas uniformemente distribuidas. Las cargas muertas y vivas uniformemente distribuidas en cada uno de los tramos tienen la misma intensidad.

- El pórtico, al cual pertenece la viga bajo análisis debe estar arriostrado lateralmente sin momentos importantes debidos al desplazamiento lateral de la estructura producido por la carga de gravedad.

\section{El método analítico utilizando el programa SAP 2000}

A fin de encontrar la autenticidad de los valores obtenidos con el método de coeficientes, se utilizará el programa SAP 2000 en cada uno de los casos que se analice según se indica en la introducción.

En cada uno de los casos mencionados, se desarrollarán las alternancias de cargas vivas para hallar los valores más desfavorables.

\section{CONFIGURACIONES, MODELOS Y} ANÁLISIS ESTRUCTURALES

3.1. Configuraciones de losas aligeradas para un centro educativo de tres pisos.

3.1.1 Losa aligerada con luces iguales.

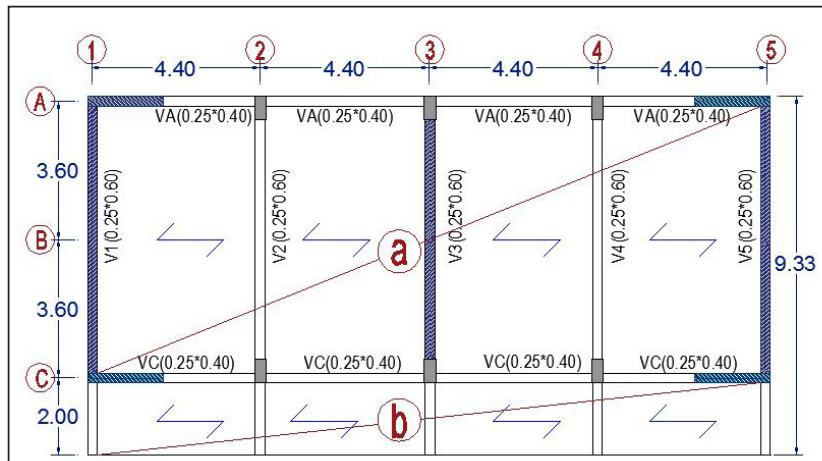

- Casos del aligerado a con apoyos combinados:

- Caso 1: Vigas y muro de albañilería artesanal

- Caso 2: Vigas y muro de albañilería Industrial

- Caso 3: Vigas y muro de concreto armado $\mathrm{e}=0.25 \mathrm{~m}$

- Caso 4: Vigas y muro de concreto armado $\mathrm{e}=0.30 \mathrm{~m}$

- Casos del aligerado $b$

- Caso 5: Todos los apoyos son vigas 
3.1.2 Losa aligerada con luces contiguas aproximadamente iguales con variaciones dentro de $20 \%$.

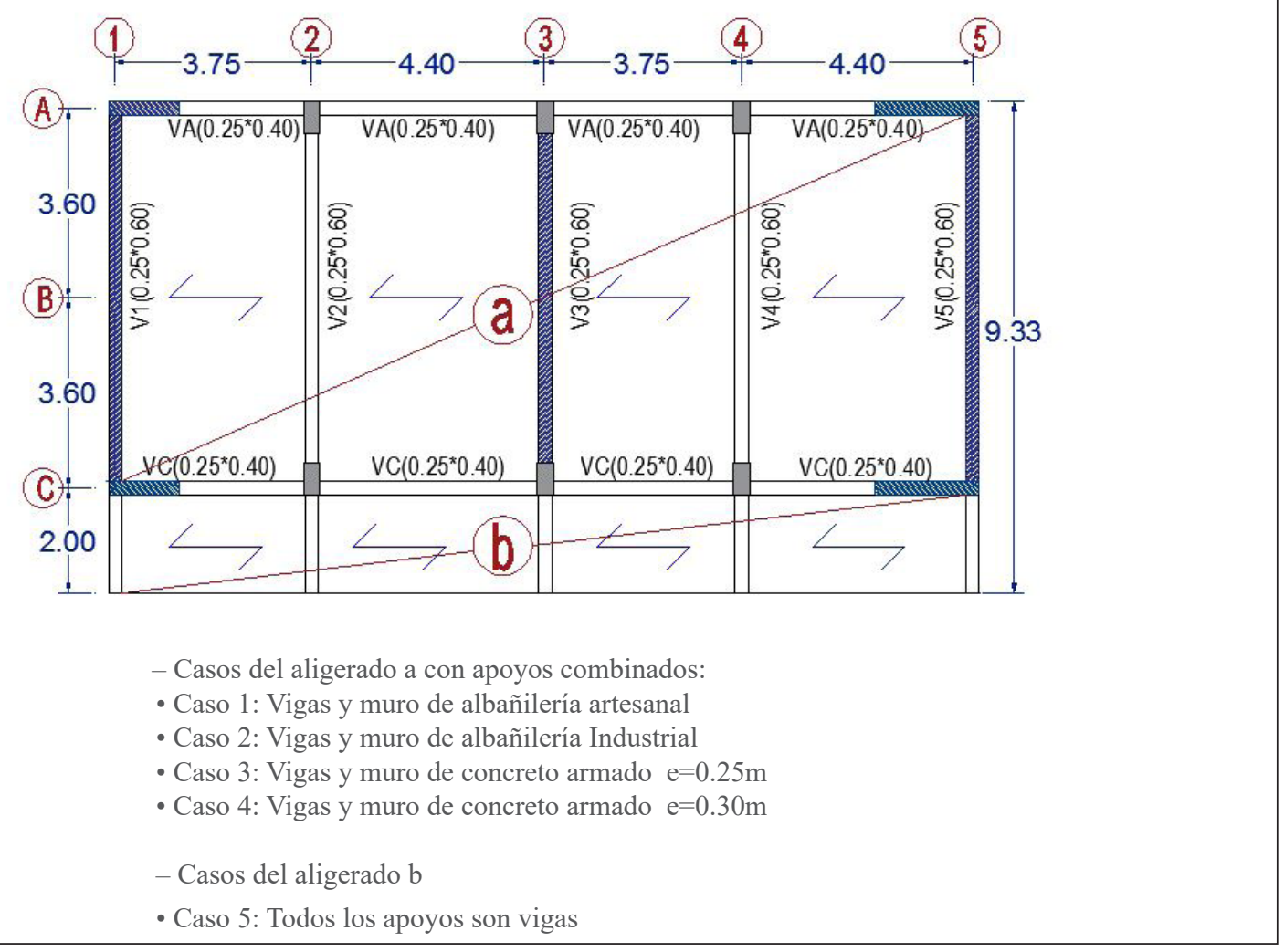

3.1.3. Losa aligerada con luces contiguas desiguales con variaciones mayores al $20 \%$.

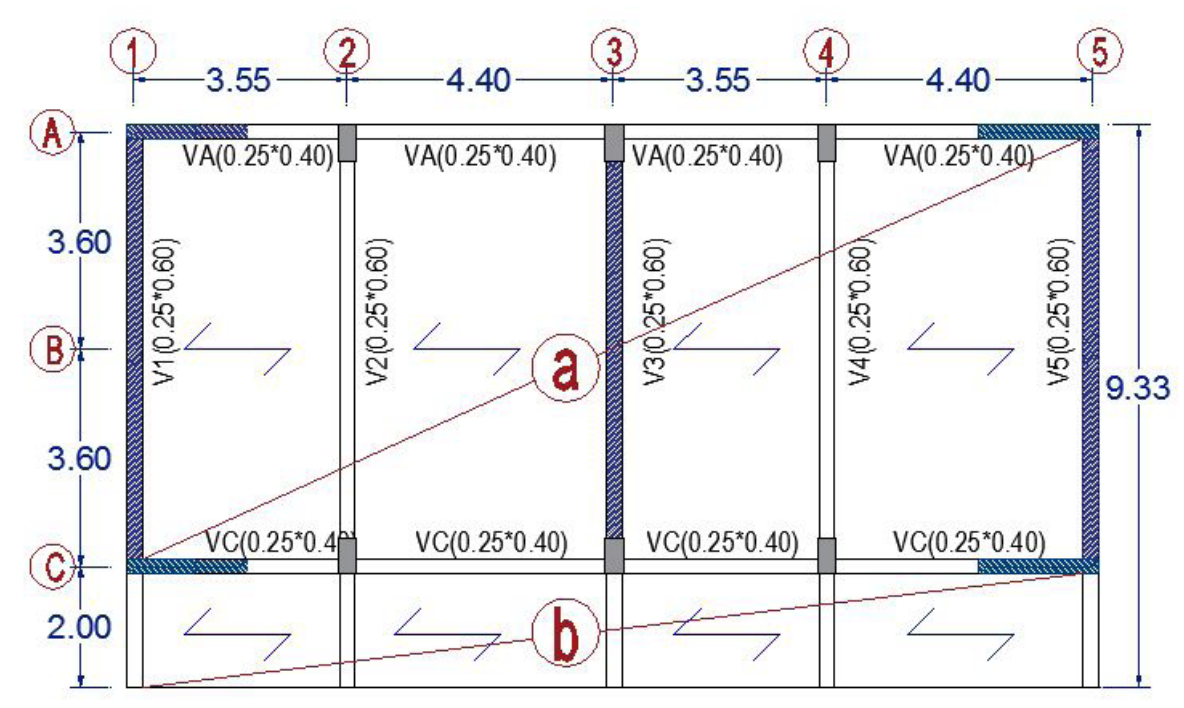

- Casos del aligerado a con apoyos combinados:

- Caso 1: Vigas y muro de albañilería artesanal

- Caso 2: Vigas y muro de albañilería Industrial

- Caso 3: Vigas y muro de concreto armado $e=0.25 \mathrm{~m}$

- Caso 4: Vigas y muro de concreto armado $\mathrm{e}=0.30 \mathrm{~m}$

- Casos del aligerado b

- Caso 5: Todos los apoyos son vigas 


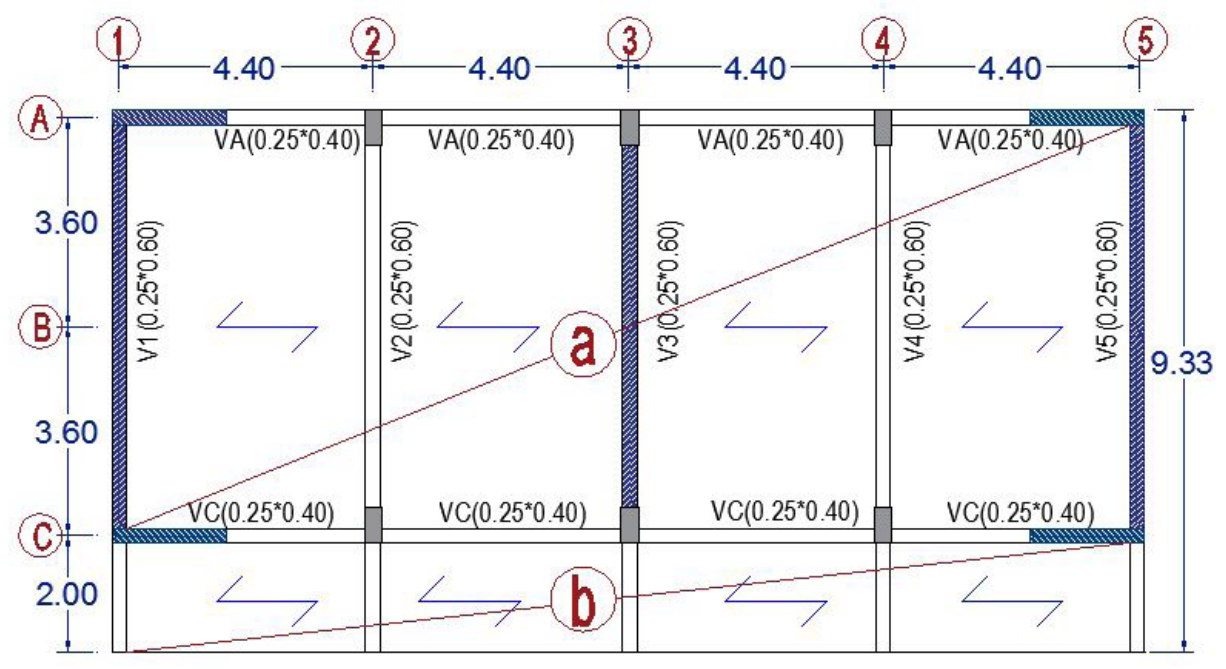

- Sólo se analiza el caso del aligerado b:

Todos los apoyos son vigas

3.2. Modelos y análisis estructurales con los métodos del ACI y el programa SAP

Leyenda

_ momentos obtenidos con el SAP

—_ momentos obtenidos con el método de coeficientes del ACI

Variables

- $\ln 1, \ln 2=\operatorname{luz}$ libre de cada tramo

- In=luz libre correspondiente al tramo

ln de tramo central=ln1o $\ln 2$

In de apoyos= promedio de luz libre de tramos contiguos

- $\mathrm{W}=$ carga última $=(1.4 \mathrm{~cm}+1.7 \mathrm{cv})$

En cada caso, la existencia de muros se presenta de dos maneras, según A. San Bartolomé [4]:

\section{Con continuidad vertical (entrepiso)}

Sin continuidad

(último piso)
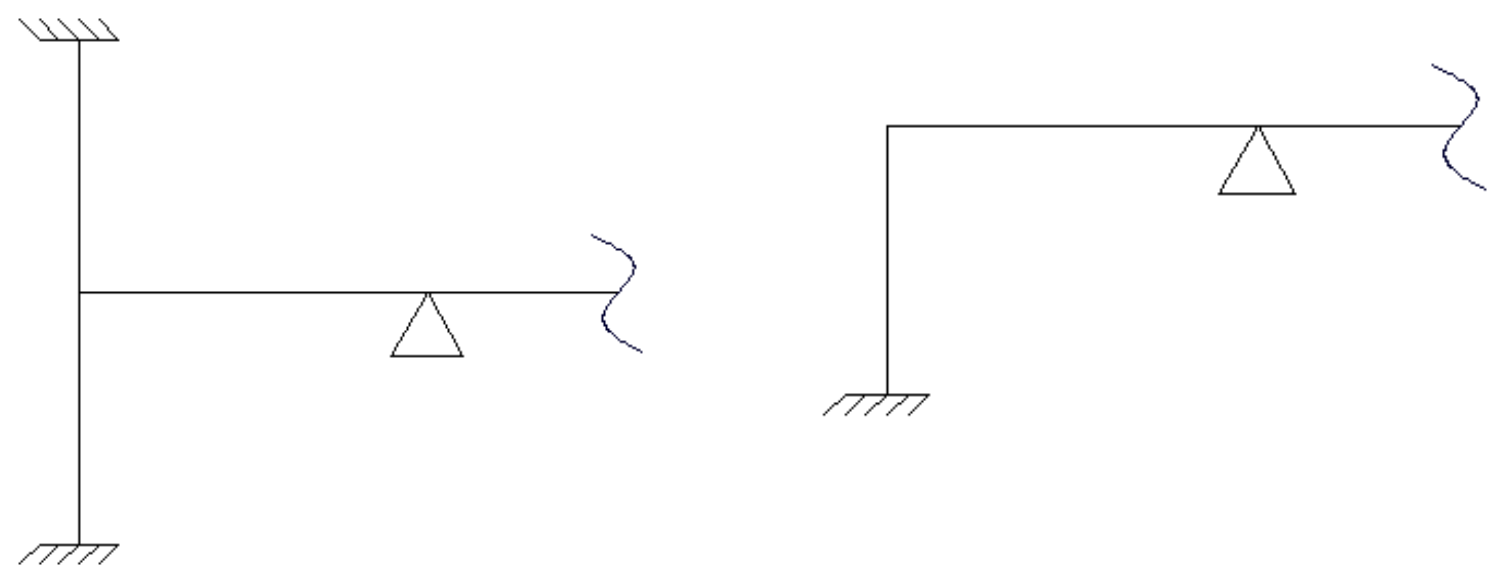
3.2.1. Losa aligerada con luces iguales

Casos

3.2.2. Losa aligerada con luces contiguas aproximadamente iguales con variaciones dentro de $20 \%$.

\begin{tabular}{|c|c|c|}
\hline Casos & Ente $p$ iso & Últipm iso \\
\hline & (1) (2) & (2) \\
\hline Caso 1 & 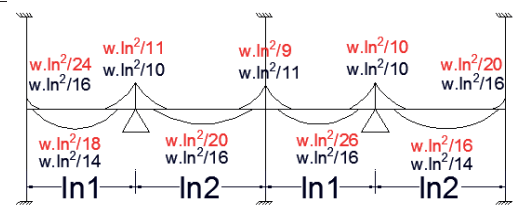 & 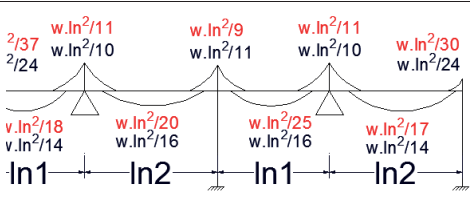 \\
\hline Caso 2 & 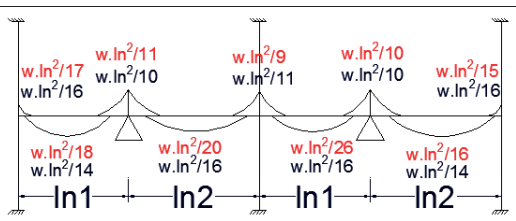 & 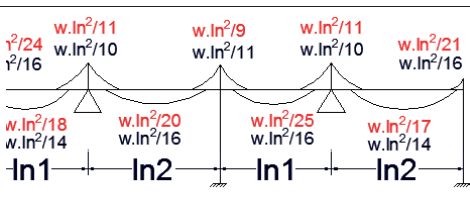 \\
\hline Caso 3 & 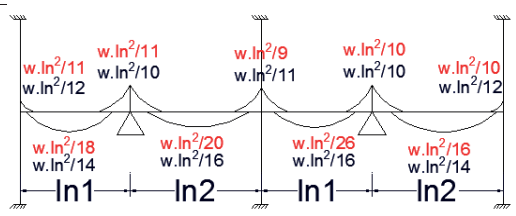 & 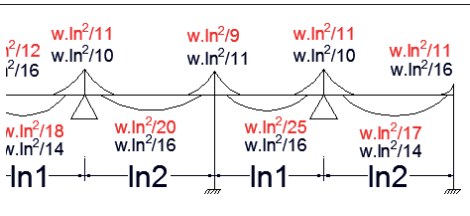 \\
\hline Caso 4 & 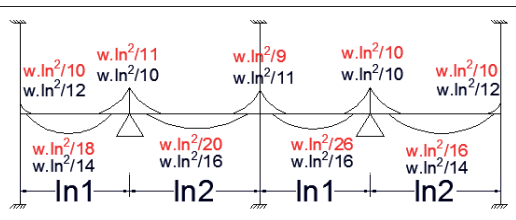 & 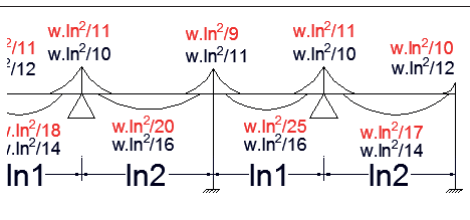 \\
\hline Caso 5 & 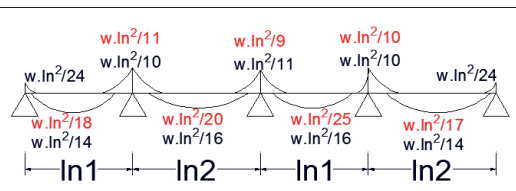 & 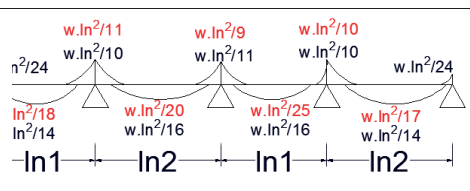 \\
\hline
\end{tabular}


3.2.3. Losa aligerada con luces contiguas desiguales

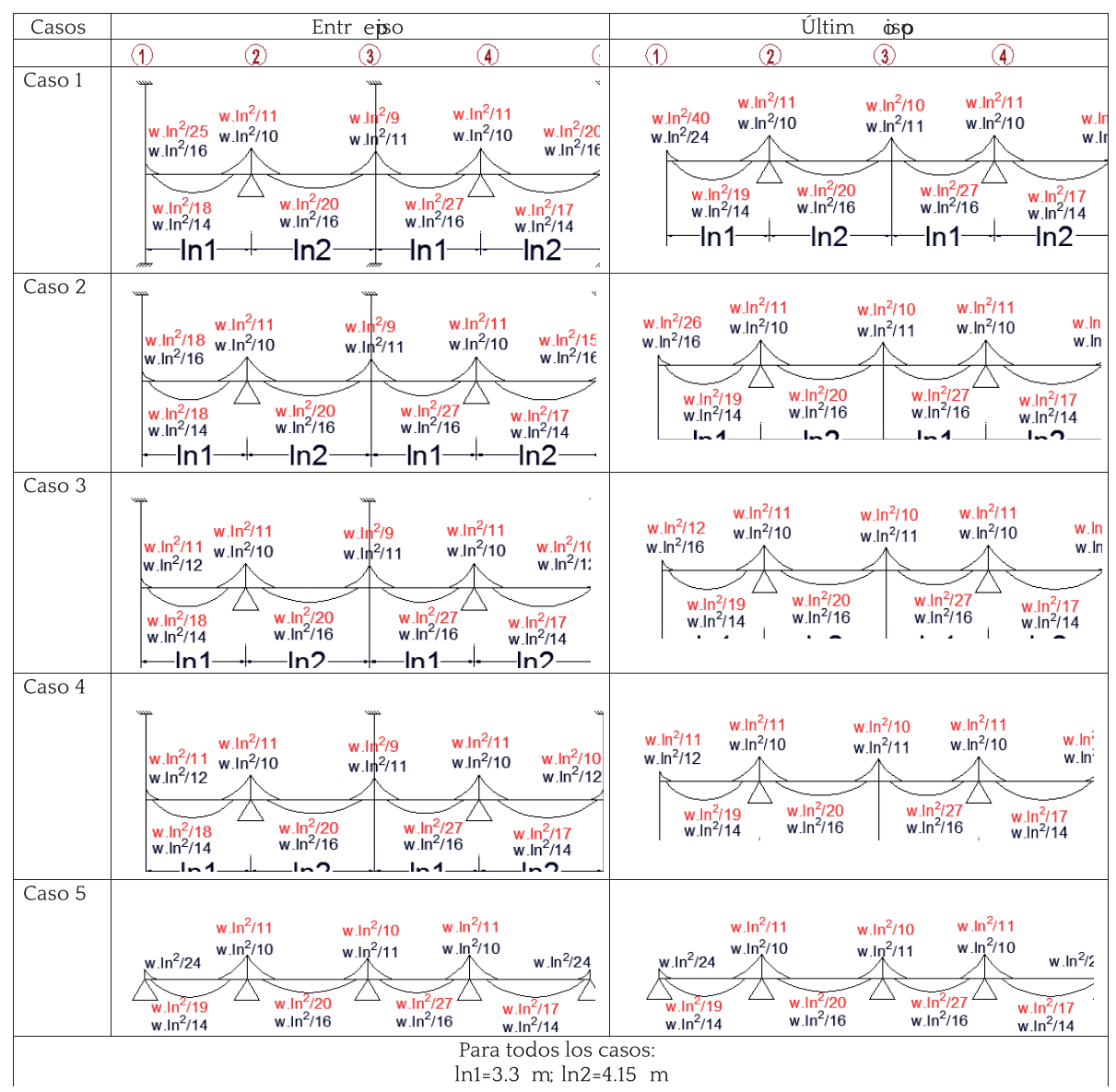

3.2.4. Losa aligerada b con luces iguales, pero con carga viva mayor que tres veces la carga muerta

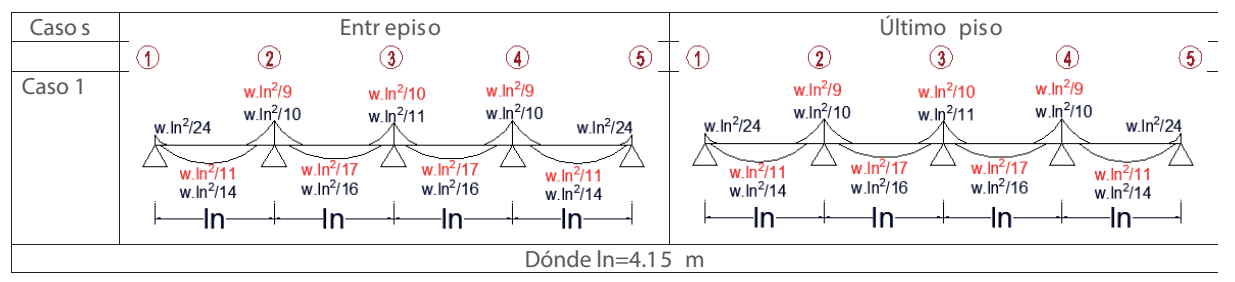

\subsection{Discusión y análisis de resultados}

De todos los análisis efectuados con el programa SAP, se deduce:

Que los momentos negativos intermedios y positivos de los tramos extremos se aproximan a resultados obtendos con el método de coeficientes del ACl

. Los momentos positivos intermedios resultan con un coeficiente promedio de $1 / 20$, mientras que el ACI especifica w.ln2/16. Sin embargo, en el análisis ejecutado para una carga cercana al límite máximo permisible por el método del ACI se llega a obtener un coeficiente muy cercano al w. $\ln 2 / 16$

- La presencia de muros en los apoyos intermedios dan resultados de momentos negativos y positivos similares a los obtenidos con apoyos vigas.

- La presencia de muros en los apoyos extremos da resultados concordantes con el ACI.

- Los momentos negativos en los apoyos extremos, G. Otazzi [3], resume de la siguiente manera:

En apoyos simples y apoyos de muros de albañilería artesanal discontinuos:

$\mathrm{w}^{*} \ln ^{2} / 24$
En apoyos de muros de albañilería artesanal continuos, albañilería industrial, concreto armado que tengan una relación de rigidez:

$$
\frac{\Sigma \text { rigideces de muros }}{\text { rigidez de viga }} \leq
$$

$\mathrm{w}^{*} \ln ^{2} / 16$

En apoyos de muros de concreto que tengan una relación de rigidez:

$$
\frac{\Sigma \text { rigideces de muros }}{\text { rigidez de viga }}>8
$$

$\mathrm{w}^{*} \ln ^{2} / 12$

Nota:
Rigide $z=\frac{E . I}{l}$; dónde:
E es módulo de elastic idad del materia ।
I es momento de inerci a de la sección
I es la luz libre del e lemento
E para la albañilería ar tesanal es $17500 \mathrm{~kg} / \mathrm{cm} \quad 2$
E para la albañilería industrial es $32500 \mathrm{~kg} / \mathrm{cm} 2$
E para el concreto (f'c $=210 \mathrm{~kg} / \mathrm{cm} 2)$ es $217371 \mathrm{~kg} / \mathrm{cm} 2$




\begin{tabular}{|c|c|c|}
\hline Casos & (1) & Leyenda \\
\hline $\begin{array}{llll}\text { Caso } & 1: & \text { Apoyos } \\
\text { combinados } & \text { de vigas y } \\
\text { muro de } & \text { albañilería } \\
\text { artesanal } & \text { en } & \text { los } \\
\text { extremos. } & & \end{array}$ & 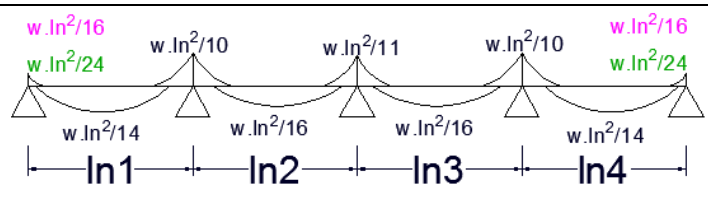 & 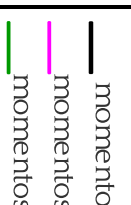 \\
\hline $\begin{array}{l}\text { Caso 2: Apoyos } \\
\text { combinados de vigas y } \\
\text { muros de albañilería } \\
\text { industrial en los } \\
\text { extremos. }\end{array}$ & $\underbrace{w \cdot \ln ^{2} / 10}_{w \cdot \ln ^{2} / 14} \underbrace{w \cdot \ln ^{2} / 16}_{w \cdot \ln ^{2} / 16}$ & 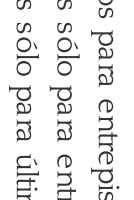 \\
\hline $\begin{array}{l}\text { Caso 3: Apoyos } \\
\text { combinados de vigas y } \\
\text { muros de concreto } \\
\text { armado e }=0.25 \text { m en } \\
\text { los extremos. }\end{array}$ & 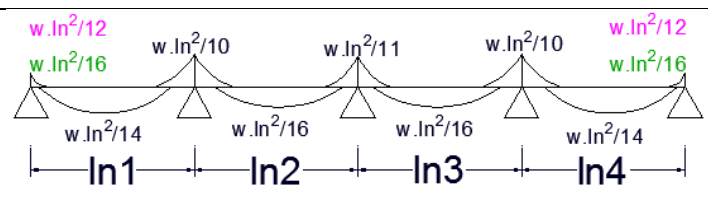 & 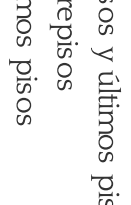 \\
\hline $\begin{array}{l}\text { Caso 4: Apoyos } \\
\text { combinados de vigas y } \\
\text { muros de concreto } \\
\text { armado e }=0.30 \mathrm{~m} \text { en } \\
\text { los extremos. }\end{array}$ & 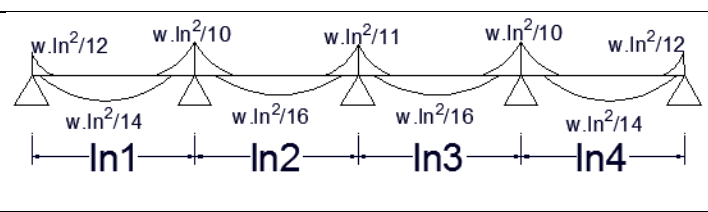 & $\begin{array}{l}n \\
0 \\
\infty\end{array}$ \\
\hline $\begin{array}{l}\text { Caso } 5: \text { Todos los } \\
\text { apoyos son vigas. }\end{array}$ & 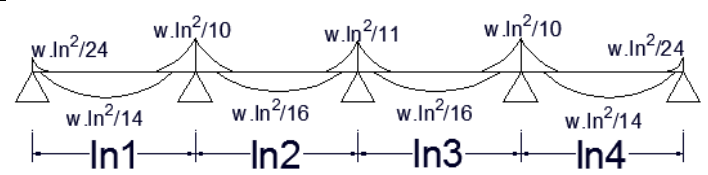 & \\
\hline \multicolumn{3}{|c|}{$\begin{array}{l}\text { - } \ln 1, \ln 2, \ln 3, \ln 4=\operatorname{luz} \text { libre de cada tramo } \\
\text { - } \ln =\operatorname{luz} \text { libre correspondiente al tramo } \\
\text { ln de tramo central= } \ln 1, \ln 2, \ln 3 \text { o } \ln 4 \\
\text { ln de apoyos= promedio de luz libre de tramos contiguos } \\
\text { - } \quad \mathrm{W}=\text { carga última= }(1.4 \mathrm{~cm}+1.7 \mathrm{cv})\end{array}$} \\
\hline
\end{tabular}

\section{CONCLUSIONES}

Para losas apoyadas totalmente en vigas y/o con apoyos extremos de muros de albañilería artesanal, los resultados obtenidos por el SAP con las alternancias de cargas vivas son:

- Para los apoyos extremos, los momentos negativos se acercan a cero. Sin embargo, se conoce que en investigaciones experimentales, efectuadas en concreto armado para vínculos monolíticos, se ha encontrado que estos toman un momento equivalente a $\mathrm{w}^{*} \ln 2 / 24$.

- Para los apoyos y tramos intermedios, los momentos positivos y negativos obtenidos son similares a aquellos obtenidos por el método de coeficientes del ACI, a excepción de los momentos positivos interiores, que son menores a los del ACI. Sin embargo, se debe aclarar que para los casos donde las cargas vivas se acercan al límite máximo permitido, según se muestra en el ítem 2.4, se cumple que el coeficiente para los momentos positivos internos es $\mathrm{w}^{*} \ln 2 / 16$.

Para las losas con apoyos combinados entre muros de albanilería industrial y de concreto armado con vigas, los resultados obtenidos por el SAP con alternancia de cargas vivas son:

Para los apoyos extremos, que son muros de albañilería confinada industrial, los momentos negativos se acercan a $w^{*} \ln 2 / 16$, resultados que concuerdan con el método del ACI.
Para los apoyos extremos, que son muros de concreto armado con espesor igual a $0.25 \mathrm{~m}$, cuando existe continuidad vertical en el muro, los momentos negativos se acercan a $w^{*} \ln 2 / 12$; pero, si el muro es discontinuo, es decir solo soporta en la parte inferior, los momentos negativos se acercan a w*ln2/16, concuerda así con el método del ACI.

- Para los apoyos extremos, que son muros de concreto armado con espesor igual a $0.30 \mathrm{~m}$, cuando existe continuidad vertical en el muro y si el muro es discontinuo, los momentos negativos se acercan a $\mathrm{w}^{*} \ln 2 / 12$, concuerda con el método del ACI.

- Para los apoyos y tramos intermedios, los momentos positivos y negativos obtenidos son similares a los obtenidos con el método de coeficientes del ACI, a excepción de los momentos positivos interiores, que son menores a los del ACI.

Cabe resaltar que para los casos donde las cargas vivas se acercan al límite máximo permitido, según se muestra en el ítem 2.4, se cumple que el coeficiente para los momentos positivos internos es $\mathrm{w}^{*} \ln 2 / 16$.

- Para las losas planteadas con luces y cargas vivas que están fuera de las limitaciones del ACI, tal como se muestra en los ítems 2.3 y 2.4, no se puede cumplir con el método del ACI. 
- De lo que antecede, se deduce que el método de coeficientes del ACI, constituye una gran herramienta alternativa para calcular momentos flectores, en forma rápida y precisa, considerando las alternancias de cargas vivas en las estructuras que están conformadas por el sistema de vigas continuas como, por ejemplo, las losas aligeradas, nervadas o macizas unidireccionales.
Según los cálculos realizados se demuestra que la existencia de muros en los apoyos solo influye en los valores de los momentos flectores en los apoyos extremos, los cuales varían de acuerdo a la relación de rigidez entre muros y vigas que conforman el vínculo.

\section{APENDICES}

\section{Apéndice 1: Alternancia de cargas vivas}

Las cargas utilizadas según el metrado son

\begin{tabular}{l|l|l}
$C M$ & 160 & $\mathrm{~kg} / \mathrm{m}$ \\
$\mathrm{CV}$ & 100 & $\mathrm{~kg} / \mathrm{m}$
\end{tabular}

El siguiente cuadro muestra los casos tomados para la alternancia de cargas vivas y su correspondiente DMF:

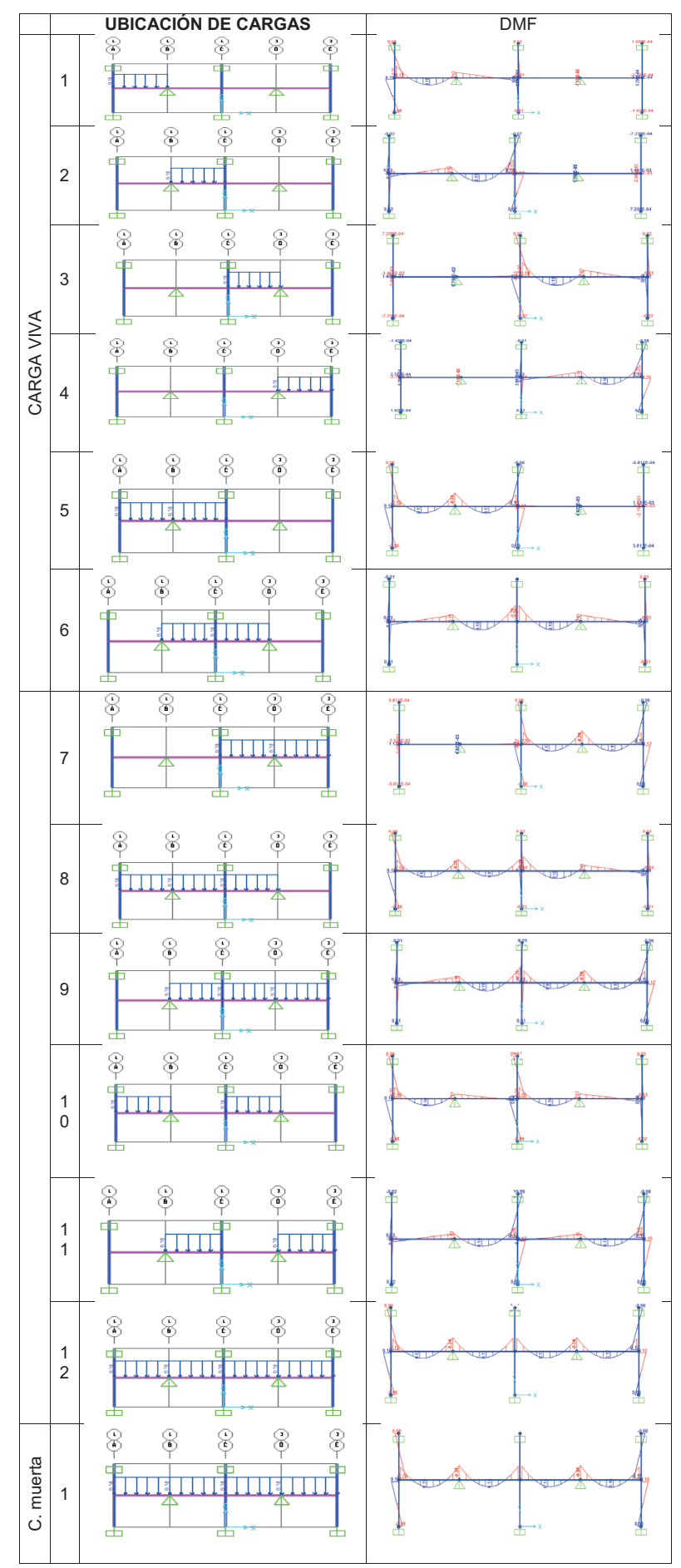


De los casos mostrados en el cuadro anterior se obtuvieron los momentos más desfavorables que resultaron de la alternancia de las cargas vivas amplificadas $\left(1.7^{*} \mathrm{CV}\right)$. Ver resultados de la envolvente mostrados con color rojo.

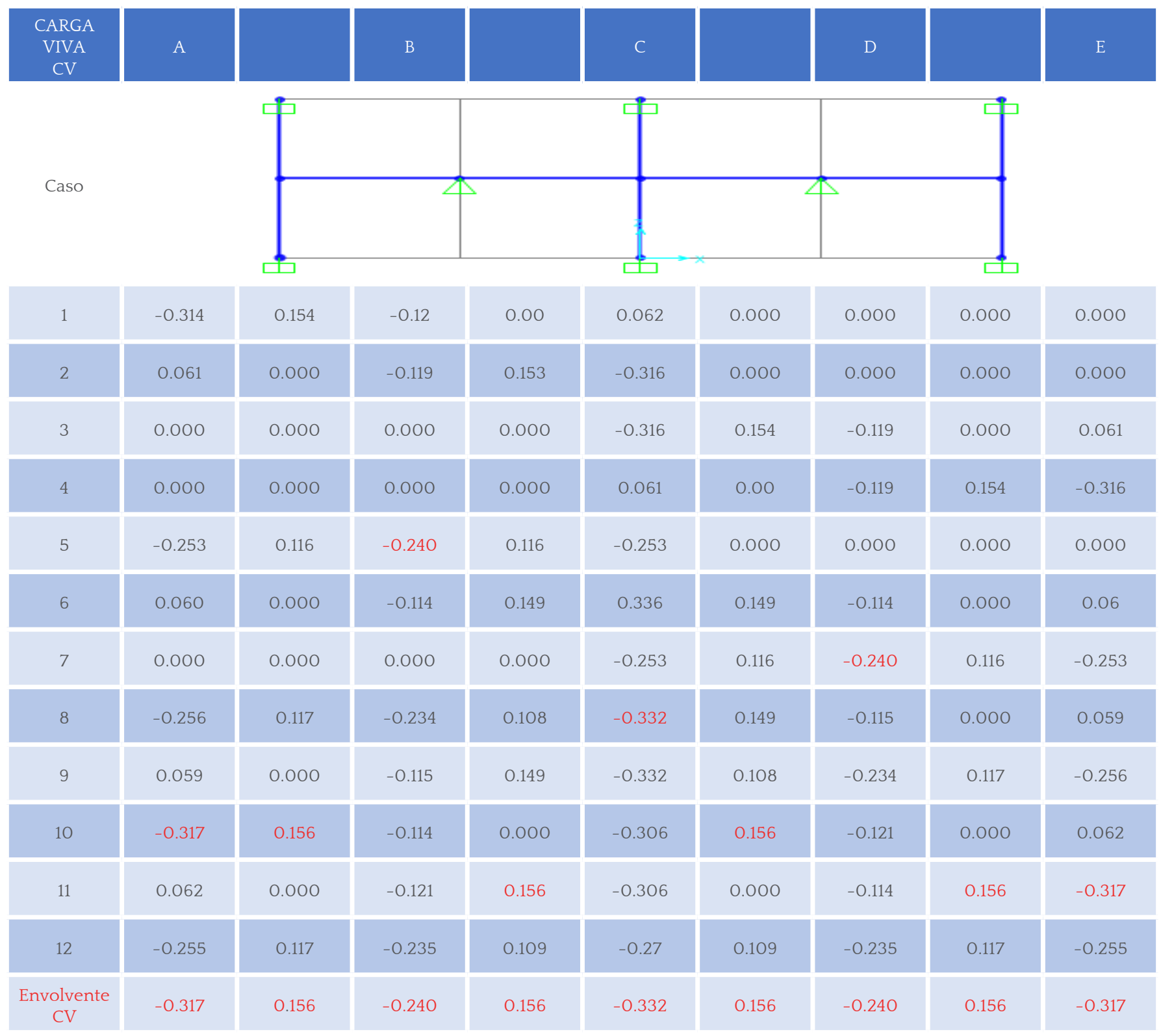

La envolvente resultante de la alternancia de carga viva amplificada debe sumarse al diagrama de momento flector de carga muerta amplificada $\left(1.4^{*} \mathrm{CM}\right)$

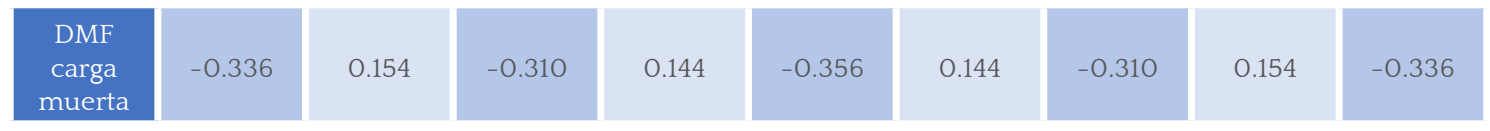

Momentos flectores resultantes de $1.4 \mathrm{CM}+1.7 \mathrm{CV}$

\begin{tabular}{|c|c|c|c|c|c|c|c|c|c|}
\hline $1.7 \mathrm{CV}$ & -0.317 & 0.156 & -0.240 & 0.156 & -0.332 & 0.156 & -0.240 & 0.156 \\
\hline $1.4 \mathrm{CM}$ & -0.336 & 0.154 & -0.310 & 0.144 & -0.356 & 0.144 & -0.310 & 0.154 \\
\hline \begin{tabular}{c|}
\hline $.4 \mathrm{CM}+$ \\
$1.7 \mathrm{CV}$
\end{tabular} & -0.653 & 0.310 & -0.550 & 0.300 & -0.688 & 0.300 & -0.550 & 0.317 \\
\hline
\end{tabular}




\section{Apéndice 2}

Relación de rigidez entre muros y vigas

$\underline{\Sigma \text { rigideces de muros }}$ rigidez de viga

Relación de rigidez en un entrepiso

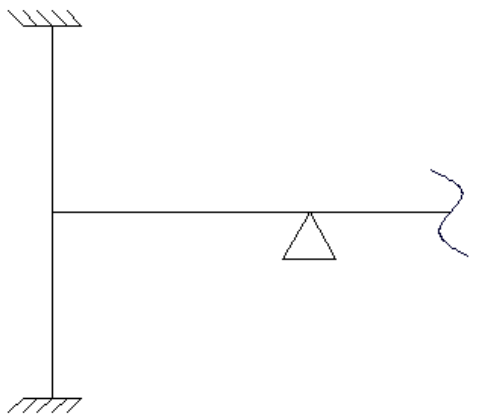

\begin{tabular}{|l|c|c|c|c|}
\hline & Caso 1 & Caso 2 & Caso 3 & Caso 4 \\
\hline Configuración 1 & 0.77 & 1.42 & 12.21 & 21.10 \\
\hline Configuración 2 & 0.65 & 1.20 & 10.30 & 17.80 \\
\hline Configuración 3 & 0.61 & 1.13 & 9.71 & 16.78 \\
\hline Configuración 4 & 0.77 & 1.42 & 12.21 & 21.10 \\
\hline
\end{tabular}

Relación de rigidez en el último piso

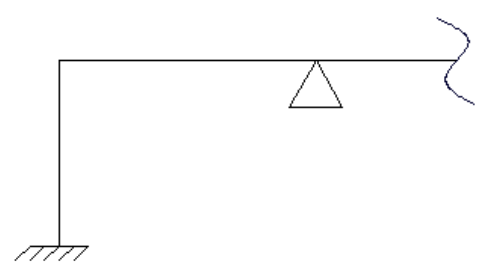

\begin{tabular}{|l|c|c|c|c|}
\hline & Caso 1 & Caso 2 & Caso 3 & Caso 4 \\
\hline Configuración 1 & 0.38 & 0.71 & 6.11 & 10.55 \\
\hline Configuración 2 & 0.32 & 0.60 & 5.15 & 8.90 \\
\hline Configuración 3 & 0.30 & 0.57 & 4.85 & 8.39 \\
\hline Configuración 4 & 0.38 & 0.71 & 6.11 & 10.55 \\
\hline
\end{tabular}

\section{AGRADECIMIENTOS}

A la Unidad de Investigación de la Facultad de Ingeniería de la Universidad Continental, por esta oportunidad.

A Kevin Lavado Sánchez por su apoyo en la traducción del resumen

\section{BIBLIOGRAFIA}

[1] Comité ACI 318, Requisitos de Reglamento para Concreto Estructural. Farmington Hills: American Concrete Institute, 2014.

[2] Ministerio de Vivienda,Norma Técnica de Concreto Armado EO.60 . Reglamento Nacional de Edificaciones. Lima: Empresa Editora Macro, 2009.

[3] G. Otazzi Pasino, Apuntes del curso concreto armado I. Lima: Fondo Editorial PUCP, 2011.

[4] Á. San Bartolomé, W. Silva y D. Quiun, Diseño y Construcción de Estructuras Sismorresistentes de Albañilería. Lima: Fondo Editorial PUCP, 2011. 